\title{
Caracterización y respuesta al tratamiento térmico de rocas silíceas opalinas
}

\author{
$M^{a}$ A. BUSTILLO ${ }^{1}$, R.GARCÍA ${ }^{1}$ Y $^{\text {a }}$ V. GARCÍA PÉREZ $^{2}$ \\ ${ }^{1}$ Dpto. de Geología. Museo Nacional de Ciencias Naturales. CSIC. 28006 Madrid. \\ ${ }^{2}$ Dpto. de Química-Física. Facultad de Químicas. Universidad Complutense. 28040 Madrid
}

\begin{abstract}
En las rocas opalinas se encuentran diferentes fases de la sílice que son poco conocidas y que ante los tratamientos térmicos responden de forma diferente. Rocas opalinas de diferentes ámbitos sedimentarios e hidrotermales se estudian mediante difracción de rayos X (DRX) y espectrometría de infrarrojos, analizando la respuesta del polvo natural y después de un tratamiento térmico a $1.050^{\circ} \mathrm{C}$ durante 24 horas. El objetivo es la diferenciación de las distintas estructuras de la sílice que pueden aparecer en las rocas opalinas, las variaciones que presentan dichas estructuras, y su cambio ante los tratamientos térmicos. En la mayoría de las muestras, la fase silícea encontrada es la denominada ópalo CT. El ópalo CT es un interestratificado de cristobalita y tridimita de estructura compleja que responde, en los análisis de DRX ,con perfiles distintos, principalmente en el intervalo de $19^{\circ}$ a $25^{\circ} 2 \theta$. Se definen tres tipos básicos en este intervalo que se denominan "perfiles ensanchados (E), de hombro $(\mathrm{H})$ y de dos picos (2P)". Estos tipos tienen relación con la proporción de tridimita en el interestratificado (va aumentando desde los perfiles ensanchados a los de dos picos), el tamaño de cristal del ópalo (los ensanchados y los de hombro tienen menor tamaño), y la reactividad ante los calentamientos a $1.050^{\circ} \mathrm{C}$ (va siendo progresivamente menor desde los perfiles ensanchados a los de dos picos). Las muestras menos reactivas resultaron ser las que poseían mayor proporción de tridimita en el interestratificado. En espectrometría de infrarrojos, los ópalos CT no manifiestan diferencias importantes entre sí, pero las muestras con perfiles ensanchados y de hombro presentan organizaciones algo distintas y mayor proporción de agua. No se han encontrado variaciones entre las estructuras de los ópalos formados en ambiente hidrotermal y los formados a temperatura ambiental, aunque en algunas muestras hidrotermales aparece ópalo C o una cristobalita $\alpha$ independiente junto al ópalo CT. Durante los tratamientos térmicos tampoco aparecieron diferencias.
\end{abstract}

Palabras clave: ópalo CT, tratamientos térmicos, DRX, espectrometria infrarrojos

\section{Characterization of opaline rocks and their behaviour UNDER HIGH temperatures}

Several silica phases are found in the opaline rocks and they show different behaviours after thermal treatments. Hydrothermal and sedimentary microcrystalline opal rocks were studied by means of X-ray diffraction and infrared absorption techniques. The measurements were carried out firstly on natural powdered samples and later on annealed samples at $1.050^{\circ} \mathrm{C}$ for 24 hours. The purpose of this study is to ascertain which silica phases appear and to evaluate what changes take place as a consequence of thermal treatments. Most of the samples are constituted of opal CT. Opal CT is a crystalline stacked sequence of tridymite and cristobalite which displays different X-ray patterns mainly in the interval from $19^{\circ}$ to $25^{\circ} 2 \theta$. Three basic types are defined according to the shape of the patterns in this interval: enlarged profiles $(\mathrm{E})$, shoulder profiles $(\mathrm{H})$ and two-peak profiles $(2 \mathrm{P})^{\prime \prime}$. These three types are related with the proportion of tridymite, the size of the opal crystals and the grade of transformation by annealing at $1.050^{\circ} \mathrm{C}$. The $\mathrm{E}$ and $\mathrm{H}$ patterns present a smaller proportion of tridymite in the stacked sequence, smaller crystals and more transformation by temperature than $2 \mathrm{P}$ patterns. The samples with less transformation after annealing are those that have more tridymite in the stacked sequence. Although the infrared absorption spectra of the opal CTs are all relatively similar, those of the E and $\mathrm{H}$ patterns present some differences in their arrangements and a higher quantity of water.

There are no differences among the structures of the opals CT formed in hydrothermal environments and those formed at environmental temperature, although in some hydrothermal samples, opal C or $\alpha$-cristobalite appears mix with the opal CT . The transformations after annealing also were similar.

Key words: opal CT, thermal treatments, X-ray powder diffraction, infrared absorption

\section{INTRODUCCION}

En las rocas opalinas aparecen diferentes fases de la sílice que son poco conocidas y que ante los tratamientos térmicos se comportan de forma diferente. Bajo la denominación genérica de ópalo se encierran diferentes fases distinguibles en DRX (Fig.1) (1) y que se denominan ópalo A, ópalo CT, y ópalo $\mathrm{C}$ donde las letras $\mathrm{A}, \mathrm{C}$ y $\mathrm{T}$, señalan la presencia de sílice amorfa, cristobalita y tridimita, respectivamente.

El ópalo A es el constituyente mayoritario de los barros siliceos formados principalmente por diatomeas, radiolarios y espículas siliceas y que son de gran interés económico $(2,3)$. Esta fase aparece también en ciertas rocas opalinas y en determinados ópalos nobles que adquieren el carácter iridiscente debido a una organización interna de las microesferas siliceas.

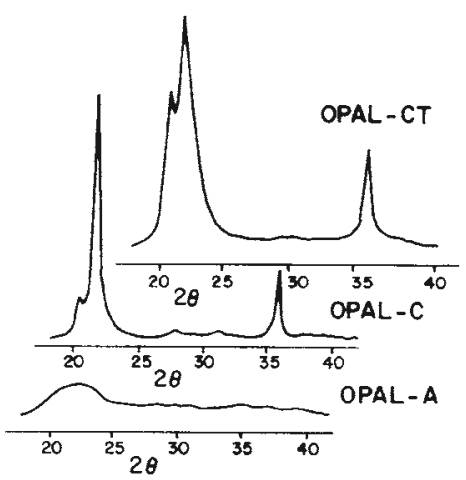

Figura 1.- Esquema de los diagramas en polvo de difracción de Rx correspondientes a las principales fases siliceas (5). 
En la actualidad esta organización ha servido como modelo para fabricar ópalos artificiales con objeto de usarlos para crear cristales fotónicos (4).

El ópalo CT se encuentra en la mayoría de las rocas opalinas compactas, bajo formas masivas y fibrosas o laminares. Es una fase compleja formada por un interestratificado de tridimita y cristobalita con distintos estados estructurales que han sido en la ultima década motivo de diferentes estudios $(5,6,7)$, determinándose incluso sus propiedades eléctricas (8). Por último el ópalo $C$ es escaso y su interés principal estriba en su valor científico en geología por ser marcador durante la diagénesis(9). Las fases mencionadas además de ser los constituyentes mayoritarios de las rocas siliceas pueden aparecer como minerales minoritarios en depósitos de interés económico tales como bentonitas, o caolines, siendo normalmente perjudiciales para sus aplicaciones industriales, por lo que es necesario su identificación y el desarrollo de técnicas de separación (10).

El objetivo de este trabajo es el estudio de diferentes rocas opalinas compactas con objeto de analizar las fases siliceas que las componen, aplicando las técnicas de difracción de rayos $X(D R X)$ y espectrometría de infrarrojos. Se intenta establecer parámetros que definan diferencias y observar su comportamiento cuando las muestras se calientan a $1.050^{\circ} \mathrm{C}$ durante 24 horas.

\section{MATERIALES Y MÉTODOS}

Se ha trabajado sobre diferentes rocas opalinas obtenidas principalmente en silcretas terciarias de las cuencas del Duero y Tajo, y en diques y venas hidrotermales de las regiones volcánicas del Cabo de Gata y Canarias (tabla I). Dichas rocas son masivas, y en ellas, el ópalo es el constituyente mayoritario, pudiendo aparecer pequeñas proporciones de arcillas, calcita y cuarzo en las de origen sedimentario y además celadonita u óxidos de hierro en las hidrotermales. Las muestras fueron escogidas en ámbitos diferentes de temperatura (sedimentarias e hidrotermales), con génesis distintas (reemplazamientos de otras rocas, transformaciones de diatomitas y rellenos de huecos) y con crecimientos cristalinos diferentes, con el fin de que muchos tipos de ópalos estuvieran representados. Se escogió, además, como ejemplo comparativo, una muestra de cristobalita de baja temperatura perteneciente a un xenolito siliceo de Lanzarote.

Para los análisis de DRX, se ha utilizado un difractómetro Philips X’PERT, dotado de monocromador curvado de $\mathrm{Cu}$, empleandose el programa PC-APD de Philips. Se realizaron diagramas sobre polvo total, en el modo de step scan, con un paso de $0.01^{\circ} 2 \theta$, y 10 segundos de tiempo. Las muestras fueron estudiadas en su polvo natural y después de un tratamiento térmico durante 24 horas a $1.050^{\circ} \mathrm{C}$, para determinar los cambios estructurales (11). Debido a que los tratamientos térmicos son esenciales, porque sirven para caracterizar las fases opalinas, basándose en la diferente reacción que manifiestan estas fases, el estudio debe realizarse sobre muestras siliceas relativamente puras. Cuando aparecen otros minerales estos interfieren, no solo por su simple respuesta en DRX, sino también, porque participan en la reacción de las fases siliceas cuando las muestras se calientan.

Los espectros de absorción infrarroja se han registrado en un equipo FTIR, Nicolet Magna 750, en el intervalo 4000-350 $\mathrm{cm}^{-1}$, con una resolución de $4 \mathrm{~cm}^{-1}$. Las muestras se prepararon como pastillas a partir de una dispersión de $0,5 \mathrm{mg}$ de producto en $200 \mathrm{mg}$ de $\mathrm{KBr}$.
TABLA 1.-LOCALIZACIÓN Y CARACTERÍSTICAS DE LAS MUESTRAS ESTUDIADAS

\begin{tabular}{|l|ll|}
\hline \multicolumn{1}{|c|}{ Localización } & \multicolumn{2}{|c|}{ Muestras / Procedencia } \\
\hline \multirow{2}{*}{ Esquivias, Toledo } & S-6 y S-16 & Niveles opalinos incluidos en diatomitas \\
\cline { 2 - 3 } & $\begin{array}{l}\text { Xil-1 } \\
\text { CXil-1 }\end{array}$ & $\begin{array}{l}\text { Xilopalo } \\
\text { Nódulo opalino circundante }\end{array}$ \\
\hline Vicálvaro, Madrid & CB-11 & Silcreta opalina \\
\hline Valdevarnés, Segovia & F-4 y CD-1 & Silcretas opalinas blancas \\
\hline $\begin{array}{l}\text { La Puna, Salar Olaroz, } \\
\text { Argentina }\end{array}$ & A-1195 & \begin{tabular}{l} 
Nódulo opalino en sedimentos terrígenos de salar \\
\hline Cabo de Gata, Almería
\end{tabular} \\
$\begin{array}{lll}\text { MQ-1, TQ-3, SG-6 Venas y diques opalinos incluidos en rocas } \\
\text { volcánicas alteradas }\end{array}$ \\
\hline Anaga, Tenerife & F-2 & Dique opalino en roca volcánica alterada \\
\hline Timanfaya, Lanzarote & XE-8 & Xenolito siliceo en colada volcánica histórica \\
\hline
\end{tabular}

\section{RESULTADOS}

\subsection{Difracción de rayos X.}

En la mayoría de las muestras se observa que la fase constituyente es el ópalo $\mathrm{CT}$ porque las reflexiones más intensas aparecen próximas a $4,11 \AA$, $4,3 \AA$, y $2,50 \AA$. No se observan las reflexiones menos intensos de $2,84 \AA$ y $3,14 \AA$ correspondientes a cristobalita $\alpha$ y que indicarían la existencia de ópalo $C(5,11)$. Sin embargo, como excepción, MQ-1 y F-2 manifiestan indicios o una pequeña reflexión $\alpha 2,84 \AA$. $\mathrm{XE}-8$ es considerada simplemente como cristobalita a ya que presenta la mayoría de las reflexiones típicas de esta fase y no aparece el ensanchamiento de la base del pico próximo $4,11 \AA$ que se da en los ópalos.

En todas las muestras con excepción de MQ-1,TQ3,CB-11 y S-6, aparecen un conjunto de reflexiones próximas a 3,9 y 3,8 $\AA$ que son asignables a los diferentes politipos de tridimita que pueden formar parte de los interestratificados $(5,8)$ y que se marcan en el perfil con pequeños hombros en el intervalo $22-23^{\circ} 2 \Theta$. Después de los calentamientos las reflexiones observadas en las muestras naturales normalmente se conservan, apareciendo como nuevas, en muchos casos, $2,84 \AA$ y $3,14 \AA$ que corresponden a la cristobalita $\alpha$ que se crea con el tratamiento térmico.

Además de estas características generales los aspectos específicos a analizar en el estudio de las fases opalinas son los siguientes:

(1) Forma del perfil de difracción en el intervalo $19-25^{\circ}$ $2 \Theta$.

Esta forma es el resultado de la variación que presenta las estructuras de ópalo CT, sobre la base de todos los factores que la determinan, siendo los más importantes: la proporción relativa de cristobalita y de tridimita dentro del interestratificado, los desordenes en los apilamientos laminares del interestratificado, el tamaño de los cristales opalinos y la proporción de sílice amorfa que puede acompañar al ópalo CT, y que modifica la forma en la base del pico. 
TABLA II Forma DEL PERFIL (ENSANCHADO (E), HOMBRO (H) Y DOS PICOS $(2 \mathrm{P}))$, POSICIÓN DE LOS PICOS $(\sim 4.11$ Y $\sim 4.3)$ QUE CONFIGURAN DICHA FORMA Y RELACIÓN DE INTENSIDADES ENTRE ELLOS.

\begin{tabular}{|l|l|c|c|c|c|c|}
\hline \multirow{2}{*}{ Muestra } & \multirow{2}{*}{ Origen } & \multirow{2}{*}{ Forma } & \multirow{2}{*}{$\begin{array}{l}\text { Espaciado } \\
\end{array}$} & & \multirow{2}{*}{$\begin{array}{l}\text { Espaciado } \\
\sim .11\end{array}$} & \multicolumn{2}{|c|}{ Relación Int c/s } \\
\cline { 5 - 7 } & & & & & $\mathrm{A}$ & B \\
\hline MQ-1 & hidrotermal & $\mathrm{E}$ & 4,074 & 4,310 & 3,87 & 5,68 \\
S-6 & sedimentaria & $\mathrm{E}$ & 4,081 & 4,310 & 1,63 & 5,41 \\
CB-11 & sedimentaria & $\mathrm{E}$ & 4,080 & 4,310 & 2,02 & 6,42 \\
TQ-3 & hidrotermal & $\mathrm{H}$ & 4,116 & 4,300 & 1,26 & 4,17 \\
S-16 & sedimentaria & $\mathrm{H}$ & 4,069 & 4,315 & 1,41 & 2,65 \\
Cxil-1 & sedimentaria & 2P & 4,119 & 4,309 & 1,53 & 2,5 \\
Xil-1 & sedimentaria & 2P & 4,096 & 4,315 & 1,79 & 2,18 \\
SG-6 & hidrotermal & 2P & 4,089 & 4,311 & 1,79 & 2,41 \\
A-1195 & sedimentaria & 2P & 4,083 & 4,300 & 1,64 & 1,4 \\
F-4 & sedimentaria & 2P & 4,101 & 4,316 & 1,66 & 1,24 \\
CD-1 & sedimentaria & 2P & 4,103 & 4,324 & 1,79 & 1,89 \\
F-2 & hidrotermal & i & 4,100 & 4,314 & 2 & 26,38 \\
XE-8 & xenolito & cristobalit & 4,043 & --- & --- & -- \\
\hline
\end{tabular}

A: Muestra en polvo

B: Después de tratamiento térmico a $1.050^{\circ} \mathrm{C}$

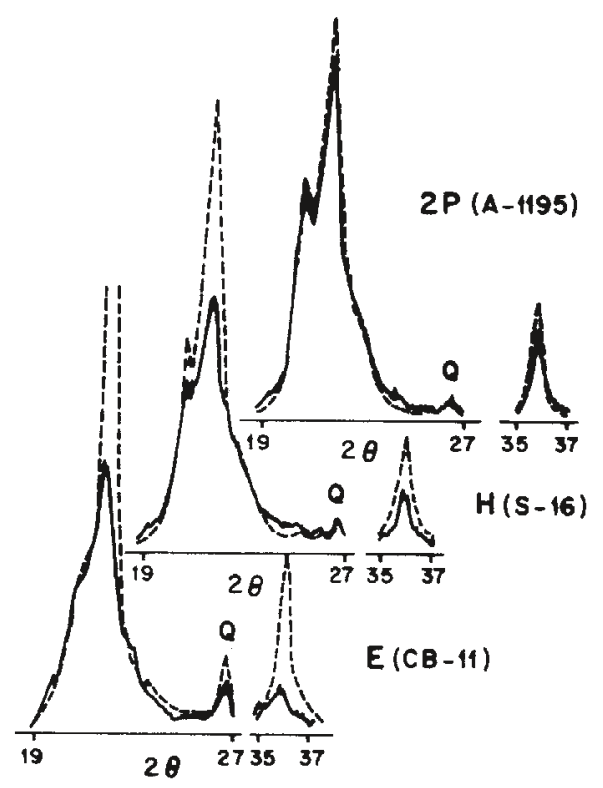

Figura 2.- Esquema de los diferentes tipos de perfiles de difracción encontrados en los ópalos.

TABLA III ESPACIADO, INTENSIDAD Y ANCHURA A MITAD DE LA ALTURA DEL PICO PRÓXIMO A 4.11Å. POSICIÓN DEL PICO SECUNDARIO.

\begin{tabular}{|c|c|c|c|c|c|c|c|}
\hline \multirow{2}{*}{ Muestra } & \multicolumn{2}{|c|}{ Espaciado } & \multicolumn{2}{|c|}{ Intensidades $\mathrm{c} / \mathrm{s}$} & \multicolumn{2}{|c|}{ FWHM } & \multirow{2}{*}{\begin{tabular}{|c|} 
Espaciado \\
$\begin{array}{c}\text { Pico } \\
\text { secundario }\end{array}$
\end{tabular}} \\
\hline & A & B & A & B & A & B & \\
\hline MQ-1 & 4,074 & 4,080 & 2.841 & 4.396 & 0,735 & 0,428 & 4,050 \\
\hline S-6 & 4,081 & 4,081 & 2.767 & 9.781 & 1,878 & 0,501 & \\
\hline CB-11 & 4,080 & 4,057 & 3.600 & 8.556 & 1,632 & 0,529 & \\
\hline TQ-3 & 4,116 & 4,053 & 2.116 & 4.251 & 2,054 & 0,654 & \\
\hline S-16 & 4,069 & 4,076 & 3.588 & 7.921 & 1,981 & 0,876 & \\
\hline Cxil-1 & 4,119 & 4,100 & 3.434 & 5.868 & 1,721 & 0,757 & 4,073 \\
\hline Xil-1 & 4,096 & 4,094 & 4.343 & 4.610 & 1,53 & 1,183 & \\
\hline SG-6 & 4,089 & 4,086 & 5.806 & 8.299 & 1,439 & 0,764 & 4,112 \\
\hline A-1195 & 4,083 & 4,115 & 5.914 & 5.746 & 1,616 & 1,292 & 4,104 \\
\hline F-4 & 4,101 & 4,095 & 5.883 & 5.256 & 1,24 & 0,911 & 4,077 \\
\hline CD-1 & 4,103 & 4,090 & 6.400 & 7.586 & 1,353 & 0,929 & 4,067 \\
\hline F-2 & 4,100 & 4,046 & 7.586 & 19.238 & 0,938 & 0,226 & 4,067 \\
\hline $\mathrm{XE}-8$ & 4,043 & 4,049 & 30.590 & 37.326 & 0,201 & 0,226 & \\
\hline
\end{tabular}

A: Muestra en polvo

B: Después de tratamiento térmico a $1.050^{\circ} \mathrm{C}$
En este estudio hemos encontrado tres tipos de forma (Fig. 2): un pico ensanchado (E), un pico con hombro (H) y un pico doble formado por dos picos bien diferenciados (2P), que se manifiestan alrededor de 4,11 $\AA$ y 4,30 Å. Estas formas no presentan relación ni con el valor de los espaciados, ni con la génesis hidrotermal o sedimentaria (tabla II). Después de calentar se definen tres comportamientos relacionados con las formas del perfil (Fig.2): a) todos los ópalos de forma ensanchada y la muestra TQ-3, cambian bastante porque cristaliza mucha cristobalita $\alpha$. En este caso, las relaciones de intensidades entre las reflexiones a $4,11 \AA$ y a $4,3 \AA$, alcanzan valores muy altos (de 4,17 a 6,42, tabla I); b) la muestra S-16, y varios ópalos de dos picos cambian también bastante aunque no cristaliza tanta cristobalita $\alpha$. Las relaciones de intensidades obtenidas entre las reflexiones referidas son mas bajas (de 2,18 a 2,65, tabla I); c) los ópalos de dos picos y que presentan gran intensidad en la reflexión próxima a $4,3 \AA$ de la tridimita (A-1195,F-4 y CD-1) no manifiestan casi cambios en la forma del perfil y las relaciones de intensidades se encuentran solo entre 1,24 y 1,89 ( tabla II). Por último la muestra F-2, presenta después de los calentamientos una transformación muy intensa y no comparable con el resto, siendo consecuencia de la presencia de celadonita (entre un 15 y un 25\%) como se expondrá posteriormente.

Estas formas de perfil tienen relación con la intensidad y anchura a mitad de la altura de la reflexión próxima a $2,50 \AA$ ( Fig.2), siendo las intensidades mas altas y las anchuras mas bajas en los perfiles de dos picos. Después de los calentamientos aumentan también las intensidades de dicho pico estrechándose su anchura en mayor medida en los perfiles ensanchados y de hombro.

\section{(2) Espaciado, intensidad y anchura a mitad de la altura del} pico próximo a $4,11 \AA$.

Este pico no suele tener valor fijo porque es consecuencia de la confluencia de las reflexiones (101) de la cristobalita y (002) de la tridimita seudohexagonal (5). En las muestras estudiadas varia entre 4,069 ̊ y 4,119 ̊, sin tener relación con el origen hidrotermal o sedimentario de los ópalos y presenta mayor intensidad en aquellas muestras de ópalo que poseen un perfil de dos picos ( tabla III). En la mayoría de las estructuras de dos picos y en MQ-1, aparece junto al máximo cercano a $4,11 \AA ̊$, otro secundario que según algunos autores sería reflejo de la confluencia de los picos de tridimita y cristobalita, no teniendo que aparecer siempre (11).

Después de los calentamientos las intensidades siempre suben bastante, excepto en las muestras A-1195 y F-4 que bajan un poco y Xil-1 que sube también un poco, indicando que estos ópalos presentan una estructura poco reactiva, ya que estas pequeñas variaciones están dentro de los márgenes de error. CD-1 aunque presenta cambio, este es pequeño, y se podria considerar también una estructura poco reactiva.

La anchura a mitad de la altura (FWHM), suele tener valores por encima de 1, siendo excepción MQ-1 y F-2 que presentan valores más bajos. Esta anchura disminuye siempre con el calentamiento, y las muestras antes definidas como poco reactivas son las que presentan después de los calentamientos mayor anchura de pico, lo que viene a indicar que han adquirido poca ordenación si se compara con los otros ópalos tratados. F-2 es una muestra que después del tratamiento térmico su anchura, que pasa a ser la más pequeña del grupo, es similar a la de la cristobalita $\alpha$ escogida como muestra de comparación. 
(3) Espaciado e intensidad del pico a 4,3 $\AA$.

La posición del pico a 4,3 Å varía poco entre las diferentes muestras ya sean sedimentarias o hidrotermales (Tabla IV) y es la reflexión indicativa de la presencia de tridimita. Al no aparecer ningún valor cercano a 4,28 ̊̊ se puede pensar que solo existe tridimita monoclínica, aunque no siempre aparecen el resto de las reflexiones. Las intensidades son variables y se relacionan con la forma del perfil de manera que los perfiles ensanchados y de hombro suelen tener los valores menores de intensidad mientras que los perfiles de dos picos manifiestan mayor número de cuentas como cabria esperar. Después de calentar, las variaciones en el espaciado y en intensidad no siguen pautas claras ya que son variaciones pequeñas. F-2, es la única que refleja un comportamiento diferente con una disminución drástica de este pico. La razón creemos que se encuentra en la interferencia que ejerce en esta reflexión la presencia de celadonita. Después de los calentamientos la celadonita desaparece y entonces el pico cercano a 4,3 $\AA$ refleja la intensidad real, que es a su vez parecida a MQ-1.

\subsection{Espectrometria de infrarrojos}

Varios espectros de infrarrojos, correspondientes a los grupos de muestras definidos por sus tranformaciones ante la temperatura (ver a,b y c,en el apartado de forma del perfil) se presentan en la Figura 3. Como se observa todos ellos son muy similares presentando bandas intensas $\mathrm{a} \approx 1100 \mathrm{~cm}^{-1}$, $\approx 790 \mathrm{~cm}^{-1} \mathrm{y} \approx 480 \mathrm{~cm}^{-1}$, asignables a vibraciones localizadas de tensión Si-O, tensión Si-Si y flexión O-Si-O, respectivamente. Las mayores diferencias entre ellos son las bandas atribuibles a vibraciones de los compuestos que en pequeña cantidad acompañan a algunos de estos materiales. Aunque no se muestra gráficamente en las muestras F-2 y MQ-1, aparecieron bandas muy débiles $\mathrm{a} \approx 620 \mathrm{~cm}^{-1} \mathrm{y}$ a unos $385 \mathrm{~cm}^{-1}$ (un hombro en el ala de bajas frecuencias de la intensa banda a $480 \mathrm{~cm}^{-1}$ ) en la muestra F-2 y a $\approx 690 \mathrm{~cm}^{-1}$ y $395 \mathrm{~cm}^{-1}$ en MQ-1, que podrían atribuirse a pequeñas cantidades de cristobalita presentes en las muestras. Como es conocido (5), estas bandas, que no suelen marcarse en el ópalo $\mathrm{CT}$, son asignables a vibraciones intertetraédricas y, por lo tanto, no aparecen cuando se pierde la periodicidad traslacional del cristal, debido a defectos en el empaquetamiento o a la dimensión excesivamente pequeña de los cristales. Una diferencia evidente entre los espectros de las muestras estudiadas se encuentra en la zona de altas frecuencias ya que se observa claramente que las bandas a $3400-3500 \mathrm{~cm}^{-1} \mathrm{y} \mathrm{a} \approx 1640 \mathrm{~cm}^{-1}$, indicativas de la presencia de agua son mucho más intensas en las muestras CB-11, S-6 y TQ-3. Esta agua proviene de la estructura opalina y de las pequeñas proporciones de arcillas que poseen dichas rocas. Por otra parte, en los espectros de esas tres muestras se observan bandas por encima de $3600 \mathrm{~cm}^{-1}$, que junto con la que aparece $\mathrm{a} \approx 950 \mathrm{~cm}^{-1}$, (hombro en el ala de bajas frecuencias de la intensa banda asignable a las tensiones $\mathrm{Si}-\mathrm{O}$ a $1100 \mathrm{~cm}^{-1}$ ), podrían ser indicativas de la presencia de grupos Si-OH. Finalmente, conviene hacer notar que un análisis en profundidad de los espectros de esas mismas muestras pone de manifiesto un ensanchamiento de la banda correspondiente a la vibración de tensión Si-Si a $\approx 800 \mathrm{~cm}^{-1}$. Este ensanchamiento de las bandas debe atribuirse a cambios en la polaridad de los enlaces debidos a modificaciones en el entorno de los tetraedros de sílice.
TABLA IV POSICIÓN E INTENSIDAD DEL PICO PRÓXIMO A $4.3 \AA$

\begin{tabular}{|l|c|c|c|c|}
\hline \multicolumn{1}{|c|}{ Muestra } & \multicolumn{2}{|c|}{ Espaciado } & \multicolumn{2}{c|}{ Intensidades c/s } \\
\hline & A & B & A & B \\
\hline MQ-1 & 4,312 & 4,306 & 734 & 773 \\
S-6 & 4,310 & 4,320 & 1.697 & 1.806 \\
CB-11 & 4,315 & 4,282 & 1.781 & 1.332 \\
TQ-3 & 4,300 & 4,285 & 1.673 & 1.018 \\
S-16 & 4,315 & 4,314 & 2.540 & 2.981 \\
Cxil-1 & 4,309 & 4,290 & 2.237 & 2.343 \\
Xil-1 & 4,315 & 4,332 & 2.421 & 2.107 \\
SG-6 & 4,311 & 4,314 & 3.226 & 3.434 \\
A-1195 & 4,300 & 4,316 & 3.600 & 4.096 \\
F-4 & 4,316 & 4,316 & 3.528 & 4.212 \\
CD-1 & 4,318 & 4,324 & 3.564 & 4.007 \\
F-2 & 4,314 & 4,280 & 3.782 & 729 \\
XE-8 & --- & -- & -- & -- \\
\hline
\end{tabular}

A: Muestra en polvo

B:Después de tratamiento térmico a $1.050^{\circ} \mathrm{C}$

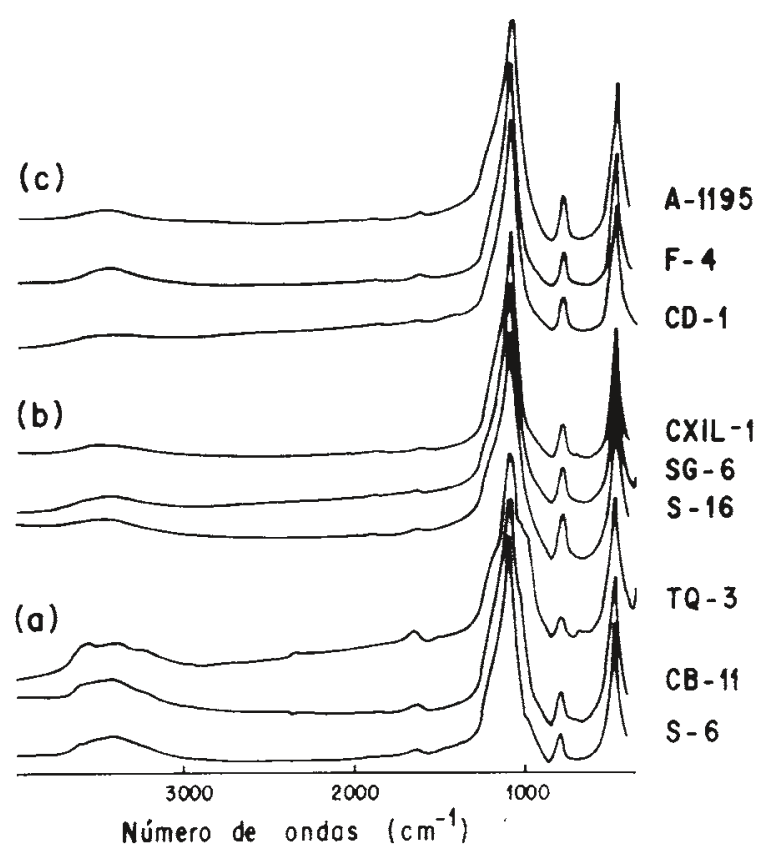

Figura 3.- Espectros de infrarrojos correspondientes a ópalos naturales que manifestaron reactividad alta (grupo a), media (grupo b) y baja (grupo c) ante los tratamientos térmicos. 


\section{DISCUSIÓN}

A pesar de la gran variabilidad encontrada en los parámetros de DRX que han sido estudiados, la mayoría de las muestras pueden ser consideradas diferentes tipos de ópalo CT. Solo dos muestras presentan características que pueden indicar la presencia de ópalo $\mathrm{C}$ o incluso la coexistencia de ópalo $\mathrm{CT}$ con una cristobalita independiente. Estas son MQ-1 y F-2, que además de tener indicios o un pequeño pico a $2,84 \AA$, presentan una anchura a mitad de la altura del pico próximo a 4,11 Å bastante mas pequeña que el resto de las muestras, siendo las relaciones de intensidades entre las reflexiones a $4,11 \AA$ y a $4,3 \AA$, altas. En el caso de F-2, además es factible que en la intensidad de la reflexión próxima a 4,3 Å este influyendo también la celadonita, ya que al calentar y destruirse este mineral, la intensidad de la reflexión cae fuertemente (tabla IV). Por lo tanto en esta muestra el valor 2 de la relación de intensidades no es real y seria mucho más alta si no hubiera esta interferencia con la celadonita. La presencia de ópalo C o cristobalita $\alpha$ sólo en F-2 y MQ-1, es corroborada en los espectros de infrarrojos, ya que las bandas débiles de cristobalita solo aparecen en estas muestras.

En los ópalos CT, los tres tipos de forma que presenta el perfil de difracción entre $19-25^{\circ} 2 \Theta$ están condicionados inicialmente por la proporción de cristobalita y tridimita dentro del interestratificado. Las formas ensanchadas, con hombro y con dos picos implican respectivamente la entrada de mayor proporción de tridimita. Este aspecto queda patente al observar la correlación que existe entre dichas formas y la intensidad (c/s) de la reflexion próxima a 4,3 $\AA$, que es la única reflexión exclusiva de la tridimita. La variación de la forma del perfil con la entrada de diferentes proporciones de tridimita ha sido modelizada en DRX considerando que a la vez que se incorpora tridimita aumenta el desorden (5), tal y como siempre se ha interpretado en el estudio de la diagénesis de rocas siliceas $(9,12)$. De acuerdos con los datos aportados en esta modelización (5) los perfiles de dos picos definidos en este estudio vendrían a tener un $50 \%$ de cristobalita y un $50 \%$ de tridimita, pero los casos de perfiles de hombro y ensanchados no quedan definidos numéricamente, aunque es evidente que existe menor proporción de tridimita. En otras ocasiones las modelizaciones en DRX son realizadas teniendo en cuenta además la regularidad del interestratificado y el tamaño de cristal de ópalo (7) determinándose que estos dos factores también afectan a la forma del perfil en este intervalo, por lo que hay que realizar un estudio general del diagrama para evaluar estos aspectos. En este sentido, la anchura a mitad de la altura del pico a 2,50 $\AA$, es sensible al tamaño del cristal del ópalo en el plano a-b (7). Teniendo en cuenta que las mayores anchuras del pico próximo a 2,50 A se han encontrado en los perfiles ensanchados y de hombro (Fig.3) hay que pensar que existe un tamaño de cristal menor en estos dos tipos.

En este estudio no se ha encontrado relación entre las formas del perfil y el valor del espaciado de la reflexión próxima a 4,11 Å. Este pico resulta de la superposición de las reflexiones de una cristobalita $(4,05 \AA$ ) y una tridimita $(4,11 \AA$ ) y puede salir entre ambos valores admitiéndose en general que a mayor proporción de tridimita se acerca a 4,11A (13). El hecho de que hayan aparecido valores mas altos de 4,11 $\AA$, ha llevado ha considerar que otros factores afectan también a este valor del espaciado encontrándose que las distorsiones estructurales en los apilamientos (5) o incluso la influencia de la materia orgánica durante la precipitación (14) alteran este valor. A su vez estudios comparados de microscopio óptico/electrónico y DRX (6) han revelado como estos valores pueden cambiar dentro de una misma muestra cuando varían las características texturales y estructurales del ópalo que la constituye. Dominios de cristobalita ordenada pueden coexistir con otros menos ordenados, presentando cada uno de ellos valores diferentes del pico próximo a $4,11 \AA$ A. De acuerdo con todos estos resultados, la ausencia de una relación directa entre los valores del espaciado, la presencia de tridimita y la forma del perfil en las muestras estudiadas es lógica ya que estos ópalos han sido formados bajo distintas condiciones y génesis (tabla I), con crecimientos cristalinos muy diferentes que han originado distintas texturas microscópicas.

Después de los calentamientos el efecto que se produce es la cristalización de una nueva cristobalita $\alpha$, que se superpone a los restos opalinos que quedan sin transformar. En general los ópalos mas desorganizados son los que manifiestan mayores transformaciones (5). En el caso estudiado las formas ensanchadas y de hombro son las que manifiestan mayores cambios, que quedan patentes en los grandes aumentos de las intensidades de las reflexiones próximas a 4,11 Å y 2,50 Å, en la disminución de sus anchuras y en la aparición de los picos menos intensos a 2,84 $\AA$ y $3,14 \AA$ y que corresponden a cristobalita $\alpha$. Considerando que el ópalo CT es definido actualmente como un interestratificado de cristobalita y tridimita en una matriz no cristalina (8), puede ser que lo que realmente cristaliza sea esta matriz que a su vez probablemente es mayor en los perfiles ensanchados y de hombro. Hay que tener en cuenta además que en los perfiles ensanchados y de hombro la proporción de agua parece mayor. Las drásticas transformaciones observadas en F-2 consideramos que son debidas a causas que están fuera de la estructura de la sílice y son ocasionadas por la celadonita. El hecho de que la celadonita desaparezca tras el calentamiento apunta la idea de que al destruirse aporta sílice que se añade a la que suministra el ópalo originándose mas cristobalita $\alpha$ de lo usual.

Los ópalos que no presentan grandes tranformaciones después de los calentamientos son principalmente los que poseen estructuras de dos picos y la proporción de tridimita es alta (intensidades superiores a 3.500 c/s en el pico próximo a $4,3 \AA$ ) por lo que hay que pensar que una mayor entrada de tridimita hace a la estructura más estable ante la temperatura. La excepción la constituye Xil-1 que es poco reactiva y no presenta valores altos de tridimita. Esta muestra es un xilópalo y ya algunos autores han advertido que estos ópalos no suelen cambiar cuando se les somete a tratamientos térmicos con temperaturas a $1.000^{\circ} \mathrm{C}(15)$. Este hecho se podría explicar considerando que en los xilópalos la materia orgánica influye en la precipitación de la sílice produciéndose estructuras de ópalo CT más organizadas (14) y menos vulnerable a la temperatura.

\section{CONCLUSIONES}

1) Los ópalos naturales suelen poseer diferentes estructuras de la sílice (ópalo A, ópalo C y ópalo CT) cuya caracterización precisa es compleja necesitándose diferentes técnicas microscópicas y analíticas. La difracción de Rx aunque presenta sus limitaciones, tal y como se ha ido exponiendo en este trabajo, sigue siendo una técnica genérica que permite un análisis global de las diferentes fases e incluso una diferenciación de tipos dentro del ópalo CT. El análisis comparativo de las intensidades y anchuras de los picos más intensos y las características generales del perfil de difracción entre las muestras naturales y las calentadas a $1.050^{\circ} \mathrm{C}$, juega un papel muy importante en las determinaciones ya que marcan que estructuras son más reactivas y que parámetros se afectan más.

2) El ópalo CT es un interestratificado de cristobalita y tridimita de estructura compleja y con muchas posibilidades de variación que responde en los análisis de difracción de $\mathrm{Rx}$ de 
forma muy diferente, originando perfiles distintos. En este estudio se han encontrado tres tipos básicos que se denominan en función de la forma del perfil en el intervalo $19-25^{\circ} 2 \theta$, como: "perfiles ensanchados, de hombro y de dos picos". Estos tipos tienen relación con la proporción de tridimita en el interestratificado (va aumentando desde los perfiles ensanchados a los de dos picos), el tamaño de cristal del ópalo (los ensanchados y de hombro tienen menor tamaño), y la reactividad ante los calentamientos a $1.050^{\circ} \mathrm{C}$ (las transformaciones van siendo progresivamente menores desde las muestras con los perfiles ensanchados a las de dos picos). La entrada de mayor cantidad de tridimita en el interestratificado proporciona mayor estabilidad de la estructura opalina ante los calentamientos, haciéndola menos reactiva.

Aunque los ópalos CT no suelen manifestar diferencias entre sí, en espectrometria de infrarrojos, las muestras con perfiles ensanchados y de hombro presentan organizaciones algo diferentes y posiblemente mayor proporción de agua.

3) No se han encontrado diferencias entre las estructuras de los ópalos CT formados en ambiente hidrotermal y los formados en ambiente sedimentario (temperatura ambiental). Sin embargo hay que señalar que en dos muestras hidrotermales aparece un ópalo C o una cristobalita a independiente junto al ópalo CT. Ante los tratamientos térmicos ópalos CT hidrotermales y ópalos CT formados a temperaturas ambientales presentan el mismo comportamiento.

\section{AGRADECIMIENTOS}

Este trabajo ha sido financiado por el proyecto PB-98-0668CO2-01 de la DGES. Nuestro agradecimiento al Servicio de
Espectroscopia de la UCM por el registro de los espectros IR. A J. Arroyo por la labor de delineación y a $\mathrm{M}^{\mathrm{a}} \mathrm{A}$. Fernandez, M. Castillejo, y J. M. Hontoria por su participación en la preparación de muestras, les agradecemos su colaboración en el trabajo.

\section{BIBLIOGRAFIA}

1. J.B. Jones y E.R. Segnit, "The nature of opal I. Nomenclature and constituent phases", Jour. of the Geological Society of Australia, 18, 57-68 (1971)

2. Ullmann, "Ullmann's Encyclopedia of Industrial Chemistry", Silica, A23, 583-660 (1993).

3. $\mathrm{M}^{\mathrm{a}}$.A. Bustillo, "Las rocas silíceas como materia prima en la industria actual", Bol. Geológico Minero, 106, 1102-1111 (1989).

4. F.J. Meseguer, C. López, H. Míguez, J. Requena y S.J. Moya, "Opalos y cristales fotónicos", Investigación y Ciencia, 269, 50-56 (1999)

5. H. Graetsch, H. Gies y I. Topalovic "NMR, XRD and IR study on microcrystalline opals", Physics and Chemistry of Minerals, 21, 166-175 (1994).

6. T. Nagase y M. Akizuki, "Texture and structure of opal-CT and opal-C in volcanic rocks", The Canadian Mineralogist, 35, 947-958 (1997)

7. G.D. Guthrie, D. Bish y R.C. Reynolds, "Modeling the X-ray diffraction pattern of opal CT", American Mineralogist, 80, 869-872 (1995).

8. L. De Pablo-Galán y L. Chávez-García y A. Huanosta Tera, "Electrical properties of opal CT", Physics and Chemistry of Minerals, 24, 90-101 (1997).

9. R. Hesse, Origin of chert: diagenesis of biogenic siliceous sediments, en Diagenesis (A Mcllreath y D.W. Morrow Eds), Geosciences Canada Reprint Series, 4, 227-252 (1990).

10. G.E., Christidis y P.W. Scott, "The origin and control of colour of white bentonites from the Aegean islands of Milos and Kimolos, Greece", Mineralium Deposita, 32, 271-279 (1997).

11. J.M. Elzea, I.E. Odom y W.J. Milnes, “Distinguishing well-ordered opal-CT and opal-C, from high-temperature cristobalite by X-ray diffraction", Analitica Chimica Acta, 286, 107-116 (1994).

12. S.B. Rice, H. Freund, W.L., Huang, J.A. Clouse y C.M. Isaacs, “Application of fourier transform infrared sprectoscopy to silica diagenesis: the opal-A to opal-CT transformation", Jour. of Sedimentary Research, 65A, 639-647 (1995)

13. J.M. Elzea y S.B. Rice, "TEM and X-ray diffraction evidence for cristobalite and tridymite stacking sequences in opal", Clays and Clay minerals, 44, 492-500 (1996).

14. N.W. Hinman, "Chemical factors influencing the rates and sequences of silica phase transitions: Effects of organic constituents", Geochemica et Cosmochimica Acta, 54, 1563-1574 (1990)

15. R.S. Mitchell y S.Tufts, "Wood Opal-A tridymite-like Mineral", American Mineralogist, 58, 717-720 (1973).

Recibido: 08.06 .99

Aceptado: 10.03 .00

\section{ENeventh $\begin{aligned} & \text { VII Conference and Exhibition } \\ & \text { of the European Ceramic Society } \\ & \text { Brujas (Belgica) } \\ & 9-13 \text { Septiembre } 2001\end{aligned}$}

\begin{tabular}{|c|c|}
\hline INSCRIPCIÓN Y ALOJAMIENTO & PROGRAMA CIENTÍFICO \\
\hline $\begin{array}{l}\text { V. de Bassompierre } \\
\text { Meeting Time }\end{array}$ & $\begin{array}{l}\text { V. Lardot- J.P. Erauw } \\
\text { Belgian Ceramic Soceity }\end{array}$ \\
\hline 13, rue du College St. Michel & venue Governeur Cornez \\
\hline B-1150 BRUXELLES- Belgium & $\begin{array}{l}\text { B-700 MONS- Belgium } \\
\text { Tel: (32) 65/50354 }\end{array}$ \\
\hline $\begin{array}{l}\text { Tele (32) } 217727393 \\
\text { Fax (32) } 2 / 7727322\end{array}$ & $\begin{array}{l}\text { Tel: (32) 65/403454 } \\
\text { Fax: (32 65/348005 }\end{array}$ \\
\hline e-mail: ecers2001@meting-time.com & e-mail: 2001@ bcrc.be \\
\hline
\end{tabular}

La European Ceramic Society fue constituida en 1987 con los objetivos de coordinar y promover los estudios e investigación sobre cerámica en Europa. Está compuesta por 20 Sociedades Nacionales y otras 8 sociedades nacionales están negociando su incorporación. Cada dos años, la ECERS organiza un congreso exposición en uno de los países miembros.

Las conferencias previas han sido organizadas con gran éxito en Maastricht (Holanda, 1989), Augsburg (Alemania 1991), Madrid (España 1993), Riccione (Italia 1995), Versalles (Francia 197) y Brighton (Inglaterra 1999).

La VII edición será organizada por la Belgian Ceramic Society y tendrá lugar en Septiembre en el Oud Sint Jan Congrescentrum de Brujas (Belgica)

El tema central serán los materiales cerámicos en su sentido más amplio desde los análisis arqueométricos, la cerámica tradicional, hasta los temas mas candentes de la cerámica avanzada. Esta sección incluirá entre otros temas como: piezas antidesgaste, componentes de alta temperatua, y materiales funcionales con propiedades eléctricas, La conferencia estará dividida en tres grandes sesiones: prón áresmiento, materiales y sesión general que incluirá métodos de caracterización y normalización. Se dedicará

asimismo un sesión especial a la Educación y Formación en Cerámica ciones, carteles y discusiones, de acuerdo con el siguiente esquema preliminar:

\section{PROCESAMIENTO}

- Polvos cerámicos

Conformado, Sinterización, Mecanizado, Tecnologías Emergentes

- Unión, Interfases, Recubrimientos

- Materiales Cerámicos con aplicaciones en Ingeniería

- Materiales Compuestos

- Electrocerámica

- Biocerámica

- Refractarios

- Cerámicas

- Vidrios, Esmaltes, Vitrocerámicos

MATERIALES CON FUNCIÓN GRADIENT

- Procesamiento, Aplicaciones en energía, m/dicina e ingeniería - Métodos de medida y caracterización

\section{SESIÓN GENERAL}

- Caracterización y Normalización

- Historia y Arque y reciclado

CONCURSOS Y PREMIOS

Premio Stuijts

Las sesiones del Congreso incluirán también la Entrega del Premio Stuijts destinado a premiar al investigador, que a juicio del Comité Director de la ECERS y de los organizadores haya hecho a lo largo de su carrera profesional una contribución especial en el campo de la Ciencau Cerámica y haya sido aplicado en beneficio de la Industria

Durante la Conferencia el Comité de Educación de ECERS organizará el Concurso a la Mejor Comunicación. Cada Sociedad Nacional elegirá el trabajo de un estudiante de su país. El elegido tendrá que presentar su trabajo, en inglés, ante un Jurado Las sesiones del Concurso estaŕn abiertas a la presencia de los congreisstas

\section{Premio al Mejor Cartel}

Los estudiantes que quieran participar deberán comunicarlo al Comité de Educación de ECERS y deberán indicarlo en el cartel. En este caso no habrá selección previa por las Sociedades Nacionales. El único requisito es que el joven investigador sea el primer firmante del trabajo y que este forme parte de su trabajo de licenciatura o de doctorado, dato que deberá ser certificado por su supervisor académico

Todos los premios serán entregados durante la cena de Clausura del Congreso

\section{FECHAS CLAVE}

31-10-2000 Fecha límite envío título y resumen

30-04-2001 Programa Provisional y envío ficha inscripción

01-05-2001 Fecha limite para enviar los trabajos completos

DATOS ENVÍO RESUMEN

Idioma Inglés

14 pts, negrilla (Centrado)

Dejar una linea entre titulo y autores

Autor (es) y afiliación. Arial 14 (Centrado)

Dejar una linea entre autores y resumen

El texto irá en Arial 12 puntos, justificado y con todos los márgenes de $2,5 \mathrm{~cm}$. El

número máximo de palabras por resumen es de 300
Enviar por correo electrónico a $2001 @$ bcrc.be 